\section{Navigating the new road in psychiatry}

\author{
By C. Simone Fishburn, Executive Editor, and Lev \\ Osherovich, Senior Writer
}

The lack of predictive animal models for neuropsychiatric diseases is arguably the biggest single factor stifling drug development in the field. To kick-start discovery for diseases such as autism, schizophrenia and depression, stakeholders will need to abandon traditional models, build on emerging genetic findings and capitalize on new capabilities in stem cell technology, imaging and computational modeling.

Ultimately, drug developers will need to come together in precompetitive consortia, share data and find consensus for new standards, techniques and models.

After numerous clinical failures, the pipeline is thin and many pharmas have stepped away from the space. But progress in basic neurological science has given rise to a new theory of synaptic connectivity as a driver of neuropsychiatric disease. ${ }^{1}$

The hypothesis posits that disorders arise from abnormalities in synaptic connections between individual neurons and between entire brain regions involved in learning, cognition and emotion.

Recent genetic studies have associated mutations in synaptic genes with autism, schizophrenia and depression. Thus, many leaders in the field now view these seemingly diverse disorders as diseases driven by alterations in brain network activity-controlled by synaptic changesand are discarding the classical view of them as neurotransmitter imbalances that can be corrected via specific receptors or transporters.

"There is a growing consensus on the study of neuropsychiatric disorders," said Mriganka Sur. "There are about 400-500 genes that, when mutated, lead to similar clinical diagnoses of autism or schizophrenia. About $70 \%$ of the genes mutated in these disorders have some kind of synaptic function." Sur is a professor of neuroscience and director of the Simons Center for the Social Brain at the Massachusetts Institute of Technology.

Although the receptor-and-transporter hypotheses of neuropsychiatric disease yielded flawed and only modestly effective drugs, they served as the premise of many animal models that became the standard for evaluation of new compounds.

For example, drugs such as $\mathrm{L}$-dopa that were effective in alleviating symptoms in patients with Parkinson's disease (PD) were introduced to animals to determine what effects they had on neurological pathways. ${ }^{2}$ Those effects then became model behavioral readouts against which researchers measured future drugs. However, the standard rodent models of neuropsychiatric disorders have not provided good predictions of how a new compound will behave in humans and are now considered to be largely irrelevant for the new targets being explored.

As genetic studies have started to reveal new targets for neuropsychiatric diseases, knockout and transgenic mouse models have begun to offer alternative ways of testing compounds. However, these too are fraught with complexities that suggest they cannot simply supplant the older models.

At the SciBX Summit on Innovation in Drug Discovery and Development in Boston, and in discussions before and after, a panel of academic and industry experts discussed the obstacles and laid out a road map for stakeholders to counter the limitations of current animal models and advance drug discovery in the field.

The panelists included Sur, Daniela Brunner, Magali Haas, Kenneth Rhodes and Mustafa Sahin.

Brunner is SVP of behavioral R\&D at PsychoGenics Inc., Haas is founder and CEO of Orion Bionetworks Inc. and Sahin is an associate professor of neurology at Boston Children's Hospital and Harvard University. At the time of the summit, Rhodes was VP of neurology research at Biogen Idec Inc. He is currently establishing a new company focused on neurodegenerative diseases.

There was wholesale agreement among the panelists that the classical behavioral models for neuropsychiatric diseases no longer serve the industry's needs.

"The old idea of studying learned helplessness in a rat and looking for drugs that change the way they fight back will not actually lead to new therapeutics," said Sur.

The think tank identified four areas that require investment and focused research: genetic linkages for neuropsychiatric diseases; new cell-based and animal models built upon molecular pathology rather than on phenotypic similarity to human disease; imaging and other technologies to study the effects of compounds on multiple brain regions; and computer modeling and network systems to integrate the information from multiple sources.

Identifying the genes that underlie the three diseases is essential for making progress and will require collaborative efforts and computerized technology to fish out meaningful results from large-scale population genetics studies.

The field should move away from thinking about animal models of disease and using behavioral readouts that aim to mimic human phenotypes. Instead, it should use animal models with relevant genetic changes and molecular pathology and should measure behavioral and other responses that indicate the target in question has been engaged. In addition, focused investment is needed in stem cell technologies to develop functional synapses in vitro.

Because autism, schizophrenia and depression involve disrupted communication between neurons and between brain regions, new ways of measuring compounds that affect those systems in intact animal and human brains are needed. That includes new imaging technologies and other noninvasive methods that can be used in both preclinical and clinical studies.

Finally, the panel advocated for investment in computerized systems that can integrate information across the different lines of evidence. In particular, it called for more public-private collaborations not only 
to share data but also to develop standards for cell-based and animal models and to drive consensus for change.

\section{Starting with genes}

The key opinion leaders agreed that the road to new models starts with identifying genes that cause synaptic dysfunction. "Genetics is the number-one stop," said Haas. "The genetic evidence for overlap between diseases is highly compelling." Indeed, one of the few areas of neuropsychiatric disease to see a surge of activity recently is large-scale genetic studies.

In July, the Psychiatric Genomics Consortium reported the results of a genomewide association study of about 37,000 schizophrenia cases and 113,000 controls. ${ }^{3}$ The consortium identified 108 loci with single nucleotide polymorphisms associated with elevated schizophrenia risk. However, no individual single nucleotide polymorphism had a strong enough effect to cause schizophrenia by itself, and the data reinforce the growing consensus that in most cases these diseases will not reduce to single-gene mutations.

Along similar lines, a team led by Joseph Buxbaum, a professor of psychiatry, neuroscience, and genetics and genomic sciences at the Icahn School of Medicine at Mount Sinai, reported in July that more than $50 \%$ of autism cases arise from the additive effect of multiple common genetic variants throughout the genome. ${ }^{4}$

The next challenge will be to parse the information yielded by these studies and identify the genes or gene combinations involved. Haas said that the best way to do this will be to use a network approach and study where mechanisms and pathways overlap and diverge. By looking at pathways disrupted in disease, researchers could reverse engineer the way back to molecules, she said.

Rhodes added that the genes identified might not be primary targets-in which the gene has a direct causal link to the disease-but could be modifier genes. Modifier genes are those found in patients who would normally get a disease but either fail to do so or develop it earlier or later than would be expected.

"Modifier genes are the next generation of drug targets. Looking for outliers in a population is what can make this go forward," said Rhodes.

Although the panelists agreed that the first step in building new models is to find the relevant targets, they also noted that using genetic information to create animal models is not straightforward. For example, Haas was skeptical that a piecemeal approach of knocking out and characterizing individual disease genes would be informative.

"These are emergent behavioral disorders with a broad range of symptoms that you won't recreate in animals," she said. "Making single point mutations and asking what happens to the animals is fun for basic scientists, but they won't get anywhere because this is not a single-gene problem."

"The idea that genetic alterations lead to a large number-perhaps a majority-of cases of neuropsychiatric disease is now widely accepted," said Sahin. "The genetics of autism are unraveling more easily than for some other diseases. But even for Mendelian disorders, we don't really have proof of principle that animal models work [like the disease] in people," he added.

Brunner was concerned that neurophysiological differences between mice and humans could complicate the interpretation of purely genetic models. For example, she said, "there are several different mouse models with different variants of Shank3 that show different behaviors and no clear rationale for choosing one model over another for drug development." SHANK3 (SH3 and multiple ankyrin repeat domains 3; PROSAP2; SPANK-2) is a postsynaptic scaffolding protein whose gene is mutated in some cases of autism.

In 2010, Buxbaum's team showed that heterozygous loss-of-function mutations in Shank3 seen in patients with autism produced only modest alterations in synaptic activity and behavior in mice. ${ }^{5}$ But in 2011, a team from Duke University showed that mice with other Shank3 mutations had severe neurological and behavioral symptoms, while a third team from The Johns Hopkins University School of Medicine showed that yet another Shank3 knockout mouse had a different set of phenotypes. ${ }^{6,7}$

Thus, for interpreting disease mechanism, "if the gene in the mouse does something different than what it does in humans, then making the same mutation in mice doesn't matter," said Brunner.

\section{Face validity vs. construct validity}

Nevertheless, the panelists said that the identification of diseaseassociated genes offers the best chance so far for moving beyond the face validity-based models that have held back drug development.

Face validity refers to models that use behaviors in animals to mimic disease-associated behavior in patients. The rationale is that if the rodent model exhibits patient-like behavior, the underlying pathological mechanisms are likely to be similar to those in humans. By contrast, construct validity refers to models based on an underlying molecular change that occurs in disease.

Sur said that excessive emphasis on face validity has misled researchers and drug makers into using models with poor predictive power. As an example, he cited anxiety disorders, which are modeled by frightening animals and testing for pharmacological interventions that alleviate fear.

"There may be some anxiety phenotypes in mice which may appear relevant to humans," said Sur. "It's likely that some complicated circuit involving the hippocampus, amygdala and frontal cortex are involved in anxiety. But many things could alter these circuits, so without construct validity from human mutations to back up the model, you have nothing."

Brunner agreed. "There is no value for face validity," she said. She had similar concerns about transgenic rodent models of autism that are based purely on behavioral abnormalities. "One popular assumption is that since people with autism have communication difficulty, we can study vocalization in mice as a model for the disease."

But she outlined how the genetic information could be used to produce meaningful models. "The first step is to determine whether 
a model has construct validity," she said. For cases in which a diseaselinked mutation appears to alter protein function, "to achieve construct validity, you need to understand which parts of the mutated protein are affected and recapitulate that in animals. Then you need to see if the pathological processes in the mouse are homologous to what you see you in humans."

However, she also said that existing behavioral assays should not be written off altogether. "There are 150 years of work on animal behavior that support many of these behavioral assays," she said. "What's important is the combination of an assay with a model that gives you predictive power."

Sur added, "The role of an animal model is to link genotype to phenotype. You would want to establish at least some behavioral or cognitive deficit. Then you would have to ask what are the plausible cellular or network phenotypes that lead to the deficit."

Rhodes said that animal behavior was useful primarily as a marker for whether a compound has affected a target.

"I agree that if you have a genetic defect, you have construct validity, but what do you measure? You still have to measure behavior, and that raises the question of whether it recapitulates what happens in people. In some ways that is not so important-as long as you have a phenotype to measure that is a readout of whether you have affected the target. We should not try to make models of disease. Instead we should look at behavior as a biomarker and not as a translational readout for what to expect in disease," said Rhodes.

He added, "The translational value is slightly shifted—but it still sets you up to design clinical trials." The data from behavioral assays "can provide tools such as pharmacokinetic/pharmacodynamic behavior that can help with clinical trial design. The most important investment you can make is in finding markers that help you know you have the right hypothesis."

Panelists agreed that there is a need for standardized methods for the construction and evaluation of genetic models. They said that there should be a battery of behavioral tests with standardized readouts and methods of validation.

One problem with defining behaviors for animal models of disease in psychiatry is that the human diseases are defined by behaviors described in the Diagnostic and Statistical Manual of Mental Disorders (DSM). The key opinion leaders agreed that DSM-defined behaviors are of limited value for drug discovery because the behaviors described in the DSM cannot be extrapolated directly to animals. Many of the definitions involve human behaviors or social interactions that have no direct parallels in animals.

"The DSM is a useful reference, but there are problems with using it to reach consensus about readouts for animal models," said Brunner.

"Basing animal models on DSM-defined disease behaviors is a very slippery slope. It still means you are trying to make inferences from the animal's state of mind to the human one. I'm not a big believer in that," said Rhodes.

\section{iPSo facto}

In addition to profiting from genomewide association studies, the field stands to benefit from new technologies that generate patient-derived induced pluripotent (iPS) cells, the panelists said. The problem is in deciding what phenotypic changes at the cellular level can be attributed to the cause of disease. In one notable case, Rett syndrome, there is good agreement between cell culture and rodent models that allows drug developers to rely on those systems for assaying candidate compounds.

Rett syndrome is a severe form of autism caused by loss-of-function mutations in methyl CpG binding protein 2 (MECP2; RTT), a DNAbinding protein. Because MECP 2 affects the expression of a large number of genes and Mecp2-mutant mice display a broad range of abnormal phenotypes, it was initially difficult to determine which phenotypes to focus on for drug discovery.

But in 2010, a team from the Salk Institute for Biological Studies generated functional neurons from iPS cells derived from patients with Rett syndrome. ${ }^{8}$ These neurons displayed a range of morphological and functional abnormalities including defects in synaptogenesis and intracellular signaling. Later studies showed that insulin-like growth factor-1 (IGF-1), which has abnormally low expression in patients and mouse models, partially restores synaptic outgrowth in cultured Rett neurons and corrects behavioral and electrophysiological defects in mouse models of the disease. ${ }^{9}$

Nonetheless, in the majority of cases in which multiple genes are involved, the absence of clear parallels between cellular and animal models makes it difficult to extrapolate from cellular abnormalities to behavioral and cognitive dysfunction.

"We have certain tests of some features of autism that work in mice, but even for the syndromic forms of disease there is still not enough validation of these models," noted Sahin.

The panelists agreed that dedicated investment is needed to show that iPS cell-based neurons can form proper synapses in a dish. A collection of patient-derived cells with robust phenotypes in vitro is a realistic goal and could be a starting point for discovery screening.

"Can patient-derived iPS cells make the right type of synapse in a dish with the right type of neurons?" asked Sur. "Even organoids have not captured synapses properly, although they have recapitulated regions and reproduced action potential firing in neurons."

Rhodes added, "It's early days for iPS cells in psychiatric diseases. We have not yet been able to get mature plastic excitatory synapses. Getting a synapse in a dish is still a key bottleneck. We need to find out what factors in the dish are needed."

Sur suggested that it would be helpful to classify patient-specific cell culture models by their distinct patterns of cellular dysfunction. He expects that, irrespective of the underlying mutations, patient-derived neurons will show defects in either excitatory or inhibitory activity.

He added that multiple types of cell-based models will be needed. "We need to make cell-specific models, but we need to know which brain regions a gene is expressed in. Only certain brain regions are associated with disease behavior," he said. "These are not disorders of just one brain system or neuron but rather of interconnectivity of brain regions. These are not behaviors that are going to be easy to model in a dish."

\section{Regional connections}

Indeed, looking at the effect of compounds on multiple brain regions is an important extension of the new way of viewing these diseases as synaptic disorders, said the panelists.

As the field's focus shifts away from individual receptors and transporters toward viewing these as disorders of connectivity, it demands exploration not only of how disease affects components within synapses but also of how it affects the way neurons connect 
and transmit information between different regions of the brain. "We need to understand the connections between brain regions to help understand these diseases," said Rhodes.

Sur said that it is impossible to predict how disease-associated changes to cellular physiology of affected neurons play out in the living brain but that starting with the phenotype makes it possible to come up with some educated guesses to guide the research.

"You would want to establish at least some behavioral or cognitive deficit. Then you would have to ask what are the plausible cellular or network phenotypes that lead to the deficit," he said.

However, the panelists agreed that the problem in the field is in deciding where to look. In addition, the field badly needs new technologies that will make it possible to better visualize deep brain regions.

Several panelists noted that functional MRI (fMRI) provides a valuable noninvasive way to image the brain but that its capabilities are limited. fMRI measures brain activity by detecting changes in blood flow, specifically by comparing oxygen-rich blood with oxygen-poor blood, based on the premise that neuronal activity requires brain cells to use energy and alters oxygenation levels and cerebral blood flow.

Rhodes also said that researchers are starting to find markers for responses to drug therapy using arterial spin labeling fMRI, a modification of the technique that avoids the need for exogenous tracers by using arterial water as an endogenous tracer. In addition, he said, diffusion tensor imaging is being used in humans for fiber tracking-a method for highlighting neurons that shows their path between different brain regions.

Rhodes added that systems neuroscience is looking at how disease affects connections between brain regions using longitudinal studies with fMRI or other imaging techniques. Brunner noted that electroencephalograms (EEGs) and some other technologies that have been around for a while have been underexploited preclinically.

In addition, there is increasing interest in drug discovery in the use of optogenetics, which uses light to activate neurons that have been engineered to express light-sensitive genes. Indeed, Circuit Therapeutics Inc. recently partnered with Boehringer Ingelheim $\mathbf{G m b H}$ to use the biotech's optogenetics technology to screen for compounds to treat psychiatric disorders. ${ }^{10}$

But none of today's techniques goes far enough, provides enough granularity or translates well between animals and humans, said the key opinion leaders.

"We need a more refined understanding of how synapses differ between brain regions and the subtle changes that occur in disease," said Sur. Little is known about synaptic differences between brain regions in either animals or humans. However, although some techniques are making headway in studying this in animals, they cannot be used in humans and thus cannot help translate from preclinical to clinical studies.

"Markers can be inserted into neurons in animals, for example, and then imaged using fMRI to light up specific individual neurons," Sur said. But what is missing, he said, are ways to do this in humans, too. Thus, what are needed are noninvasive technologies that could work in humans. Better fMRI is one solution, but there are concrete limits to what any single technique can do, he said. "At this point, we can't introduce extrinsic markers into humans-so we're left with only intrinsic signals that can be measured."

Brunner agreed that improved imaging technologies in nonhuman primates could help in looking for a signature that would define the response.

She noted that bioinformatics is already being used to look for behavioral signatures associated with drugs and mutations. For example, PsychoGenics has developed SmartCube, one of three proprietary behavioral systems used to phenotype models of autism and other disorders. The 'cubes' are high throughput, in vivo platforms that involve computer vision to automatically capture rich behavioral datasets covering many different behavioral domains. These datasets are used to train machine-learning algorithms to define drug or mutant signatures.

\section{From imaging to the big picture}

Bioinformatics, big data and large-scale collaborative projects are perhaps the cornerstones of the next phase in translational research for all areas of neurology.

For example, the NIH BRAIN (Brain Research through Advancing Innovative Neurotechnologies) initiative aims to use advanced microscopy to map functional circuits in the entire brain, starting with simple animal models such as flies and worms. ${ }^{11}$

But for neuropsychiatric diseases in particular, the panel was unanimous in calling for collaborative public-private initiatives to advance technologies, share information, create consensus for how animal models should be implemented and change accepted standards for what preclinical data are required to move to the clinic.

Haas noted that the Institute of Medicine has taken steps in this direction with a workshop held in 2012 on improving translation of animal models for nervous system disorders. ${ }^{12}$

She added that public-private partnerships using computational approaches will be needed to make sense of the volumes of genetic information coming out of genomewide association studies. In addition, she said, "what could help drive change would be to gather large amounts of human data via precompetitive consortia to serve as reference sets that could be used to inform animal models."

Rhodes agreed. "Ultimately this is the only way that this will be done. The more partnerships for sharing data in an open and transparent way, the better off for everyone. We are making investments in large computational modeling [at Biogen Idec], but even there it is only looking at a small piece of the pie. People sharing data openly is key to success moving forward."

The same is true for animal models, said Sahin. He noted that the notfor-profit organization Autism Speaks is forming a consortium of labs for unifying models across different laboratories. The need for not-for-profit organizations to drive this is huge, he said. "Without such support, there is little incentive for an academic lab to do this."

Rhodes and Haas noted that companies could help_or even leadrather than walking away from the field.

"Biotechs are pulling out rather than doubling down," Rhodes said. "Some of the big players who have had a lot of revenue have pulled away. They haven't just walked away; it's been kind of an Olympic sprint." 


\section{ANALYSIS}

Haas added, "Industry needs to define the translational zone. The challenge is that academia has produced siloed endeavors. Pharma needs to rethink the investment model. Recently they have moved to external innovation, but they need to invest in precompetitive collaborations. So far industry has been more willing to move to a model of open science than academics."

Haas proposed a Framingham-type study that would involve accumulation of observational data in longitudinal studies in a defined population. The study would be a modern-day version that would include deep and frequent genotyping of a group of people, in addition to characterizing elements such as cognitive performance, nutrition, environmental factors and microbiome status to find what factors correlate with the development of psychiatric disease in that population.

But she questioned whether the current approach should be rethought altogether. "The overreliance on models may be limiting us," she said. "We need to take a new look at first in human studies and ask, "What evidence do we really need? Must it be an animal model'?"

Either way, the field needs to devise readouts that are clinically meaningful and to engage the FDA in the process, Rhodes said.

"Companies need to make every effort to work closely with regulators to develop novel and more meaningful endpoints, particularly in disease indications where it has been notoriously difficult to develop new therapies. Sometimes this means running expensive clinical studies using endpoints that are not well validated, which can be very challenging, and some companies have more of a stomach for it than others," he said.

Ultimately, the panelists said, there is no alternative to finding new ways of measuring the effects of candidate compounds, and precompetitive consortia offer the best option for exploring the available possibilities and fleshing out the details. Investors, industry and regulators will need to get on board if those new ways are to translate to anything meaningful for patients.

Fishburn, C.S. \& Osherovich, L. SciBX 7(31); doi:10.1038/scibx.2014.913 Published online Aug. 14, 2014

\section{REFERENCES}

1. Fishburn, C.S. \& Osherovich, L. SciBX 6(41); doi:10.1038/scibx.2013.1153

2. Hornykiewicz, O. J. Neurol. 257, S249-S252 (2010)

3. Schizophrenia Working Group of the Psychiatric Genomics Consortium. Nature 511, 421-427 (2014)

4. Gaugler, T. et al. Nat. Genet. 46, 881-885 (2014)

5. Bozdagi, O. et al. Mol. Autism 1, 15; published online Dec. 17, 2010; doi:10.1186/2040-2392-1-15

6. Peça, J. et al. Nature 472, 437-442 (2011)

7. Bangash, M.A. et al. Cell 145, 758-772 (2011)

8. Marchetto, M.C.N. et al. Cell 143, 527-539 (2010)

9. Castro, J. et al. Proc. Natl. Acad. Sci. USA 111, 9941-9946 (2014)

10. Cukier-Meisner, E. BioCentury 22(1), A18-19; Jan 6, 2014

11. Osherovich, L. \& Lou, K.-J. SciBX 7(24); doi:10.1038/scibx.2014.692

12. Institute of Medicine. Improving the utility and translation of animal models for nervous system disorders: workshop summary. (The National Academies Press, 2013)

\section{COMPANIES AND INSTITUTIONS MENTIONED}

Autism Speaks, New York, N.Y.

Biogen Idec Inc. (NASDAQ:BIIB), Weston, Mass.

Boehringer Ingelheim GmbH, Ingelheim, Germany

Boston Children's Hospital, Boston, Mass.

Circuit Therapeutics Inc., Menlo Park, Calif.

Duke University, Durham, N.C.

Food and Drug Administration, Silver Spring, Md.

Harvard University, Cambridge, Mass.

Icahn School of Medicine at Mount Sinai, New York, N.Y.

Institute of Medicine, Washington D.C.

The Johns Hopkins University School of Medicine,

Baltimore, Md.

Massachusetts Institute of Technology, Cambridge, Mass.

National Institutes of Health, Bethesda, Md.

Orion Bionetworks Inc., Cambridge, Mass.

PsychoGenics Inc., Tarrytown, N.Y.

Salk Institute for Biological Studies, La Jolla, Calif. 